\title{
Les « Mots de l'histoire » un moment fédérateur de la recherche franco-allemande en histoire
}

\section{Pierre Monnet}

\section{OpenEdition}

\section{Journals}

Édition électronique

URL : http://journals.openedition.org/ifha/462

DOI : $10.4000 /$ ifha. 462

ISSN : 2198-8943

\section{Éditeur}

IFRA - Institut franco-allemand (sciences historiques et sociales)

\section{Édition imprimée}

Date de publication : 30 septembre 2012

Pagination : 195-203

ISSN : 2190-0078

\section{Référence électronique}

Pierre Monnet, «Les « Mots de l'histoire » un moment fédérateur de la recherche franco-allemande en histoire », Revue de l'IFHA [En ligne], 4 | 2012, mis en ligne le 14 février 2013, consulté le 03 mai 2019. URL : http://journals.openedition.org/ifha/462 ; DOI : 10.4000/ifha.462

Ce document a été généré automatiquement le 3 mai 2019

(CIFHA 


\title{
Les « Mots de l'histoire » un moment fédérateur de la recherche franco-allemande en histoire
}

\author{
Pierre Monnet
}

1 Comme tout objet scientifique ou sujet d'étude, ce séminaire peut, et même sans doute doit se prêter à une opération d'historicisation. Lorsque le séminaire a démarré en 2004, la réforme des universités françaises n'avait pas encore commencé, l'Université francoallemande (UFA) mettait à peine en place quelques outils de soutien à la recherche entre la France et l'Allemagne, les collèges doctoraux franco-allemands (CDFA) n'existaient pas encore, la cotutelle restait encore une procédure complexe et peu répandue, le Centre interdisciplinaire d'études et de recherche sur l'Allemagne (CIERA) avait 3 ans d'existence, la Mission Historique Française en Allemagne (MHFA) était encore à Göttingen et l'on ne parlait pas encore d'un Institut français d'histoire en Allemagne (IFHA, à Francfort seulement depuis 2009), le Centre Marc Bloch (CMB) avait encore ses locaux au Schiffbauerdamm...

2 Huit ans plus tard, bien des changements ont affecté le paysage universitaire et scientifique de part et d'autre du Rhin, les organisateurs historiques du séminaire ont pour beaucoup changé d'affectation ou de statut, la France et l'Allemagne ont procédé chacune à leur manière à leur initiative d'excellence, modifié leurs procédures d'évaluation et de financement de la recherche sur projets, la MHFA a déménagé à Francfort et est entrée dans un partenariat serré avec son université d'accueil, la Goethe Universität, de même que le Centre Marc Bloch changeait de locaux et entrait en partenariat conventionné avec la Humboldt Universität, tandis que l'Institut historique allemand de Paris (IHAP) qui nous accueille aujourd'hui changeait de directeur et modifiait en profondeur son profil scientifique. L'UFA développait dans le même temps une ambitieuse politique de soutien à la jeune recherche, multipliait les cotutelles devenues plus faciles à conclure, lançait l'appel d'offres à la création de CDFA, soutenait davantage de cursus intégrés en sciences sociales et humaines. 
3 Pendant tout ce temps, le séminaire des « Mots » conservait sans mutation majeure la forme et le principe de ses travaux, mais se faisait aussi le témoin et l'acteur des changements évoqués. C'est donc sur ce double constat qu'on souhaiterait faire reposer cette brève réflexion consacrée à la manière dont les « Mots » ont constitué un moment de fédération, une étape du maillage scientifique franco-allemand : à savoir une pérennité de forme et de formule d'un côté mais en même temps l'adaptation à une accélération des réformes et des remembrements du paysage scientifique et académique de l'autre, vérifiant en quelque sorte la célèbre phrase prononcée par Tancredi au prince Salina dans le Guépard de Lampedusa, pour qui « Il faut que rien ne change pour que tout change ». Il fallait en effet tout d'abord que rien ne change dans la formule des « Mots » pour qu'elle soit en mesure d'accueillir d'année en année de nouveaux partenaires, de nouvelles institutions, de nouvelles universités par le biais des intervenants. Au total, du côté des participants allemands ou européens, ce sont 34 universités différentes qui ont été représentées, parmi lesquelles Berlin arrive en tête avec les trois établissements de la capitale, la Humboldt Universität (3), la Freie Universität (2) et la Technische Universität (1) ainsi que le Wissenschaftszentrum Berlin WZB (1), soit 7 au total, suivie de Göttingen, Munich et Constance avec chaque fois quatre orateurs, puis Bielefeld, Dresde et Francfortsur-le-Main avec 3 intervenants, puis Francfort-sur-l'Oder, Sarrebruck, Düsseldorf, Cologne et Münster avec 2 invités. Viennent ensuite, avec chaque fois un intervenant, Greifswald, Trèves, Bochum, Darmstadt, Eichstätt, Stuttgart, Essen, Erfurt, Erlangen, Hambourg, Heidelberg, Braunschweig et Augsbourg. En Suisse, Bâle, Berne et Lucerne ont adressé 5 intervenants tout ensemble. Au-delà, comptons Rotterdam, Cambridge et Tel Aviv.

5 Ce sont tout d'abord les absents qui peuvent retenir l'attention dans cette carte des correspondants de la République humaniste des "Mots». Tout d'abord l'Autriche en qualité de pays germanophone, mais dont il est vrai les universités, faute de relais sur place sous la forme d'un institut scientifique français ou d'un Frankreichzentrum, ne sont que sporadiquement et non structurellement tournées vers la recherche française. Pour l'Allemagne même, l'Est et le Nord sont moindrement représentés, tandis qu'à l'ouest et au sud, font défaut Tübingen, Bonn, Hanovre, Kassel, Ratisbonne, Ulm, Würzburg. Cette cartographie des lieux allemands touchés par l'exercice de comparaison historiographique et méthodologique proposé par le séminaire fournit plusieurs enseignements. Tout d'abord, la présence de la MHFA à Göttingen puis de l'IFHA à Francfort explique qu'avec 7 orateurs provenant de ces deux villes, ce centre a bien fait fonction de relais et de plate-forme de dialogue scientifique franco-allemand. Cette remarque vaut également pour le Centre Marc Bloch et les institutions berlinoises qui ont fourni, elles aussi, un contingent comparable de 7 intervenants, soit un total de 14 participants sur 51 collègues allemands convoqués, c'est-à-dire une proportion de quelque $30 \%$. Cette « pole position » berolino-göttingo-francfortoise se trouve en quelque sorte renforcée par l'existence depuis 2006 d'un collège doctoral franco-allemand entre l'EHESS et la Humboldt Universität et depuis 2009 entre Paris I et l'université de Francfort, l'un et l'autre placés sous le toit de l'UFA. Parmi les autres fournisseurs allemands des « Mots ", la présence de Dresde tient à l'existence d'une ancienne coopération doctorale de cette université avec l'EPHE et plus généralement avec les collègues historiens de Paris, celle de Sarrebruck par un tropisme franco-orienté traditionnel de cette université et de son centre d'études françaises, que soutiennent le siège de l'UFA depuis 1999 dans cette ville et l'activité de 3 cursus intégrés franco-allemands de Sarrebruck avec Metz (en 
SHS et formation des enseignants). L'existence de cursus intégrés franco-allemands de l'UFA en histoire renforce également la présence de Bielefeld (cursus avec Paris VII), mais ici un dialogue scientifique en SHS de longue durée existait entre l'école de Bielefeld et l'EHESS et la MSH), de Bochum (cursus avec Tours) et de Heidelberg (cursus avec l'EHESS) dans ce panorama. L'absence d'un maillon du cursus intégré en histoire entre Tübingen et Aix-en-Provence et entre Dijon et Mayence vaut d'être notée. Quant à Münster, les liens scientifiques avec l'historiographie française se renforcent depuis la mise en place d'un centre d'études françaises depuis 2 ans dans cette université.

6 À cela pourrait s'ajouter le rôle joué par les Frankreichzentren dans la structuration de ces échanges : si celui-ci est avéré dans le cas du centre de la FU de Berlin, mais aussi sans doute de Sarrebruck, il est bien moins avéré dans le cas de Leipzig et de Fribourg, absentes de la liste des lieux centraux "christallériens » des " Mots", tout comme est absent l'institut franco-allemand de Ludwigsburg.

7 L'entrée plus résolue du DHIP depuis 2008 dans l'organisation et la conception du séminaire, particulièrement à la faveur d'un changement de direction, se manifeste par la participation de sept chercheurs relevant de cette institution au séminaire, preuve du rôle occupé par cet établissement dans l'animation du dialogue scientifique francoallemand en histoire et en SHS plus largement.

Bien entendu, ces raisons d'ordre structurel n'excluent en rien le jeu des relations et des complicités personnelles entre les animateurs du séminaire et leurs partenaires allemands, pas plus qu'elles ne font l'économie des choix dictés par les thématiques et les compétences mobilisées par les concepteurs du séminaire pour faire appel aux spécialistes de telle ou telle entrée retenue pour une séance. De la même façon, l'analyse doit prendre en compte le chevelu des croisements et des coopérations favorisé et entretenu par les programmes de mobilité et de bourses des institutions actives dans ce segment : le CMB, la MHFA et l'IFHA, l'IHAP, le CIERA, la MSH et son programme francoallemand, l'UFA, sans oublier au cas par cas le DAAD.

9 Malgré l'absence du côté allemand d'une structure comparable au CIERA du côté français assurant une fonction de fédération organisée des études allemandes sur la France, la contribution germanique aux « Mots de l'histoire » reflète la géographie des points forts par lesquels passe outre-Rhin l'échange scientifique avec les chercheurs et universitaires français, en détachant des réseaux qui prennent appui sur l'action des deux centres de recherche français en Allemagne, le CMB à Berlin, et la MHFA de Göttingen puis l'IFHA de Francfort, qui reposent aussi sur l'existence de collèges doctoraux franco-allemands et de cursus intégrés de niveau master.

$\mathrm{Du}$ côté français, la répartition de l'origine des intervenants reflète le paysage différemment polarisé de la recherche sur l'Allemagne ou en lien avec l'Allemagne. Là où l'enquête du côté allemand faisait certes apparaître quelques concentrations mais renvoyait toutefois l'image d'une répartition plus harmonieuse sur l'ensemble du territoire, fédéralisme oblige, la carte française souligne de nouveau un effet manifeste de polarisation sur le môle parisien. L'EHESS fournit, de loin, le plus gros contingent des intervenants (les chiffres reposant sur un dénombrement du nombre d'occurrences et non du nombre de personnes, un même collègue de l'EHESS par exemple peut être comptabilisé plusieurs fois) avec 33 places occupées au cours des séances des 8 années passées. Si l'on ajoute 8 intervenants inscrits au CRIA, 13 à Paris I, 3 dans les deux Écoles Normales Supérieures (ENS) parisiennes (Ulm, Cachan), 2 à Sciences Po Paris, 3 à Paris IV, 3 à l'Institut d'histoire du temps présent (IHTP), 3 à Paris VII et 2 à l'École pratique des 
hautes études (EPHE), 1 à Paris VIII, 1 à Paris X, 1 à Paris XIII et 2 à l'UVSQ (Versailles/ Saint-Quentin-en-Yvelines), et 4 dans les laboratoires parisiens du CNRS, le pôle parisien occupe à lui seul près de $90 \%$ des places, ne laissant à Lyon que 3 entrées (ENS et Sciences Po), et ensuite à Angers, Brest, Mulhouse, Amiens et Clermont-Ferrand, avec respectivement un intervenant, un reliquat très maigre. Deux facteurs peuvent expliquer une surreprésentation aussi écrasante, même au regard du poids de Paris dans l'ensemble du dispositif de la recherche française. La localisation géographique du séminaire tout d'abord, qui s'est toujours tenu à l'EHESS, au CIERA puis à l'IHAP. Le rôle fédérateur du CIERA ensuite, qui orchestre depuis Paris l'ensemble de la fédération des études françaises sur l'Allemagne. À quoi il convient d'ajouter que, du côté français, les concepteurs du séminaire relevaient tous d'institutions parisiennes : EHESS, CRIA, IHAP, universités de Paris I, Paris IV, Paris VII. D'autre part, les deux collèges doctoraux francoallemands soutenus par l'UFA et très investis dans la tenue du séminaire impliquent d'un côté l'EHESS avec la Humboldt Universität de Berlin et de l'autre Paris I avec la Goethe Universität de Francfort. Enfin, pour achever ce tableau, les cursus intégrés francoallemands en histoire soutenus également par l'UFA et mobilisés par le séminaire unissent Paris VII et Bielefeld, Paris IV et Bonn, l'EHESS et Heidelberg.

11 Cette concentration parisienne ne saurait en rien préjuger d'une hiérarchie géographique de l'excellence scientifique, elle traduit bien davantage la réalité d'une densité capitale et traditionnelle des institutions d'enseignement supérieur et de recherche dont le maillage a été précisément structuré et développé par la création du CIERA il y a maintenant plus de 10 ans. Elle n'appauvrit nullement le réservoir de ressources mises à contribution au service du séminaire et place le CIERA et son réseau en position d'interlocuteur privilégié pour des partenaires allemands intéressés par un dialogue scientifique, dont la diversité n'est pas tourmentée par l'existence de cette plate-forme, même si son fonctionnement, on y reviendra, rend aujourd'hui sans doute souhaitable l'existence d'un CIERA allemand ou en tout cas d'une structure apte de l'autre côté du Rhin à fédérer et à coordonner les études germaniques sur la France.

12 Cette cartographie succincte des liaisons nouées pendant les huit éditions annuelles du séminaire des « Mots » met au jour un réseau dont la vitalité ne repose pas seulement sur les échanges et pérégrinations portés par des collègues en poste, mais qui s'anime aussi par une mobilité incitant les étudiants de master et de doctorat à circuler d'un pays à l'autre. C'est bien entendu aussi pour ce public que le séminaire des «Mots » a été en dernier lieu fondé, dans l'objectif affiché non seulement d'enrichir différemment la formation des jeunes chercheurs en les rompant aux méthodes de la comparaison, des transferts, de la traduction et de la conceptualisation interculturelle, mais aussi avec l'ambition de constituer en amont la génération des prochains acteurs du dialogue scientifique franco-allemand. On a pu de ce point de vue souligner à raison le rôle que jouent les cursus intégrés et les collèges doctoraux franco-allemands soutenus par l'UFA et impliqués dès l'origine dans la tenue du séminaire. C'est bien là l'effet visé par une politique de développement des outils de formation bilatérale et bi-diplômante et de soutien à la recherche poursuivie par cet organisme, et qui compte aujourd'hui 125 cursus intégrés dont 33 en SHS (parmi lesquels on en dénombre 9 en histoire, 6 en sciences politiques et 8 en études franco-allemandes), et 28 CDFA dont 7 en SHS, quelque 150 cotutelles en cours, une cinquantaine de manifestations sous la forme d'écoles d'été, de dialogues scientifiques interculturels et d'ateliers de recherche engageant environ 1500 
participants au cours d'une seule année. Beaucoup sont des étudiants et des doctorants encadrés par les organisateurs et les participants du séminaire.

Mais au-delà, l'intégration d'étudiants, de doctorants et de jeunes chercheurs au séminaire est également le produit d'une politique de soutien à la mobilité orchestrée par les programmes de bourses mises à disposition par les organismes co-porteurs du séminaire. Il suffit de citer les 50 doctorants impliqués dans les programmes de formation et de recherche conduits par le Centre Marc Bloch, à quoi s'ajoute la douzaine de boursiers soutenus par ce centre et l'école d'été. Du côté du CIERA, c'est un volant comparable de quarante boursiers soutenus que l'on peut citer en 2011, en ajoutant les 189 participants au programme de formation doctorale, les 21 participants au séminaire des jeunes chercheurs du Moulin d'Andé et les 125 mastérants inscrits dans le programme master. Du côté de l'IHAP, mentionnons les 17 boursiers soutenus pour l'année académique 2010-2011, les 27 participants à l'école de recherche d'automne et les 33 participants de l'école d'été. Concernant l'IFHA, ce sont en moyenne 30 à 40 boursiers de courte durée qui sont soutenus chaque année pour effectuer une mobilité en Allemagne. Si l'on regarde leur établissement d'inscription, on s'aperçoit qu'environ la moitié d'entre eux, ainsi 12 sur 27 en 2011, proviennent du réseau des organisateurs du séminaire ou des cursus ou collèges doctoraux franco-allemands du premier périmètre (voir le bilan de 35 années de bourses à l'IFHA/MHFA dressé dans le présent numéro de la Revue).

Tout compte fait, le séminaire constitue bien assurément à la fois un vecteur et un support des échanges, mobilités, synergies établis entre des institutions qui structurent la circulation, la formation et la production des étudiants, jeunes chercheurs et chercheurs confirmés sur l'histoire allemande, l'histoire française, l'histoire franco-allemande ou l'histoire européenne conçue dans une interdisciplinarité des SHS et dans une approche comparée des notions et des méthodes. À sa manière, le séminaire a formé une étape indispensable sur la voie d'une structuration de la recherche mais aussi d'une pratique plus intégrée de la recherche dont témoignent par ailleurs les deux entreprises parallèlement conduites dans le même temps du manuel d'histoire franco-allemand et de la collection de la Deutsch-Französische Geschichte en 11 volumes publiée sous l'égide de l'IHAP et présentée dans les colonnes de la présente Revue. Les " Mots » s'insèrent bien dans un appareil et un réseau du dialogue scientifique franco-allemand en SHS, qui croise le travail pérenne d'instituts sédentaires de recherche, qui intègre l'apport d'outils limités dans le temps que sont les cursus binationaux, les collèges doctoraux francoallemands et les écoles d'été et qui prend appui sur une formation et une mobilité organisées dès le master par le biais des bourses.

Les organisateurs du séminaire, cela a été dit dans les interventions précédentes, avaient volontairement conçu cette manifestation non comme une fin en soi, mais comme une forme expérimentale de la recherche, comme une pratique partagée entre historiens français et allemands afin de mettre au jour dans les traditions et les méthodes les ressemblances, les convergences, les divergences et les transferts autour d'une notion en usage dans leur communauté respective. Il n'est donc pas étonnant que cette expérience et cette pratique aient engendré un autre projet, que beaucoup ici connaissent déjà et par lequel il convient d'achever ce propos. Il s'agit du projet « Saisir l'Europe : un défi pour les sciences sociales et humaines" (également présenté dans ce numéro de la Revue). Ce projet est lié de deux manières au séminaire des « Mots ». D'un point de vue scientifique d'abord, sa thématique se situe dans le prolongement du travail de comparaison notionnelle entamé voilà huit ans dans la mesure où, face à l'ébranlement des modèles 
sociaux, politiques et économiques provoqués par la crise, les SHS cherchent à trouver un discours moins explicatif et rétrospectif que prospectif pour tenter de penser à nouveaux frais les catégories et les méthodes de saisie et d'interprétation des liens et formes d'organisation territoriale et sociale qui ont été jusqu'à présent étiquetés comme une spécificité proprement européenne, mais partant plurielle, d'un modèle étatique social, d'un type de développement et enfin d'une manière de résolution des conflits. Dans cette opération d'interrogation conceptuelle et méthodologique, nourrie des exigences de la profondeur historique, de la pluridisciplinarité, du plurilinguisme et de l'interculturalité, il a paru opportun de capitaliser l'expérience acquise au sein du séminaire. Du point de vue organisationnel en second lieu, ce projet est également lié au séminaire puisque l'on retrouve pour l'animer les grandes institutions porteuses du séminaire : le CMB, l'IFHA, le DHIP, la MSH et les deux universités allemandes partenaires du CMB, la Humboldt Universität, et de l'IFHA, la Goethe Universität, l'une et l'autre ne se trouvant pas par hasard impliquées dans un collège doctoral franco-allemand. De la sorte, ce projet «Saisir l'Europe », présenté lors du 4e Forum franco-allemand de la recherche tenu à Berlin en octobre 2011 puis intégré en février 2012 par les deux ministères français et allemand de la recherche parmi les huit projets pilotes d'un agenda scientifique stratégique commun pour les huit prochaines années, approfondira le travail de maillage institutionnel déjà mis au jour pour ce bilan de huit années des "Mots», et finalement conservera et enrichira l'esprit même qui a soufflé sur les séances depuis 2004-2005, puisqu'il s'agit bien de croiser au fond une double herméneutique, celle d'un objet d'une part, et celle de la manière de comprendre cet objet d'autre part, tout en prenant acte d'une nouvelle pratique de la jeune recherche, qui saisit l'Europe à la mesure même dont elle la parcourt physiquement et par-delà les frontières. Cette mobilité, comme on l'a souvent vu pendant les séances, fait bouger les étudiants et les collègues tout en faisant bouger les frontières disciplinaires, méthodologiques et lexicales. C'est bien dans ce sens, et fort du savoir-faire acquis pendant le séminaire, que les institutions porteuses du nouveau projet «Saisir l'Europe » ont construit un fonctionnement en réseau autour de trois axes et de trois sous-projets, chacun associant des post-doctorants et des doctorants des deux pays, l'objectif du réseau ainsi constitue étant aussi, comme pour le séminaire des "Mots", celui de la formation à la recherche par la recherche.

C'est sur cette note optimiste, puisqu'aussi bien le BMBF allemand a décidé le 1er juin 2012 de financer la conduite de ce projet pour une durée de cinq ans par une enveloppe financière conséquente, aussitôt suivi par son homologue français le MESR, qu'il convient de terminer, en soulignant combien le modèle du séminaire des "Mots », et combien la complicité logistique et intellectuelle tissée entre les institutions organisatrices, ont abouti à une nouvelle étape dans la structuration et le rapprochement des sciences sociales et humaines entre les deux pays et leurs communautés scientifiques : il faut bien, n'est-ce pas, que tout change pour que rien ne change. 


\section{AUTEUR}

\section{PIERRE MONNET}

Pierre Monnet est directeur de l'IFHA. 